\title{
Kernos
}

Revue internationale et pluridisciplinaire de religion grecque antique

$21 \mid 2008$

Varia

\section{Synnøve DES BOUVRIE (éd.), Myth and Symbol II. Symbolic phenomena in ancient Greek culture}

\section{Ellen Van Keer}

\section{OpenEdition}

Journals

\section{Édition électronique}

URL : https://journals.openedition.org/kernos/1707

DOI : 10.4000/kernos. 1707

ISSN : 2034-7871

\section{Éditeur}

Centre international d'étude de la religion grecque antique

\section{Édition imprimée}

Date de publication : 1 janvier 2008

Pagination : $352-355$

ISSN : 0776-3824

Référence électronique

Ellen Van Keer, «Synnøve des bouvrie (éd.), Myth and Symbol II. Symbolic phenomena in ancient Greek culture », Kernos [En ligne], 21 | 2008, mis en ligne le 15 septembre 2011, consulté le 24 août 2022. URL : http://journals.openedition.org/kernos/1707 ; DOI : https://doi.org/10.4000/kernos.1707 
Fallait-il donc cette entrée en matière jargonnante (parmi les nouveaux incontournables, on relèvera plus loin, pour faire pendant à «naitre », l'élégant « n'être »...) et un discours de la méthode si assuré et parfois si méprisant qui se prétend quant à lui innocent de tout «arraisonnement» du mythe ? Car on apprendra encore, dès le début du premier chapitre, que « la simplicité » de Chaos a été « jugée, comme telle, irrecevable par les interprètes dont la raison d'être complique à souhait ce que peut bien vouloir dire ce mot neutre ». Bref, on l'a échappé belle !

Ces remarques un peu sévères n'entendent pas discréditer tout l'ouvrage. L'auteur maitrise bien son sujet et la longue revue critique, savamment ordonnée, des multiples exégèses du Chaos hésiodique, impressionne par son érudition et par un art subtil de l'argumentation. Ce qui en est dit ensuite positivement permet assurément de mieux comprendre ce que l'introduction énonçait de façon assez énigmatique. Il n'est pas sûr cependant que cette exégèse soit appelée à évincer définitivement les autres. M'est avis qu'elle prête à Hésiode plus et plus compliqué qu'il n'a pensé et, en tout cas, qu'il n'a dit. Le texte du poète apparait plus d'une fois sollicité. Du Chaos il est dit, par exemple, qu'il est né (egenet) en premier; Hésiode n'indique pas, il est vrai, que Chaos engendre Gaia, mais pour celle-ci, alors que le même mot egenet' est sous-entendu, on ne dit plus qu'elle est née, mais qu'elle «advient». Du Chaos on affirme aussi , à plusieurs reprises, qu'il "pousse », et même qu'il " pousse les choses à devenir ", que "sa naissance pousse au dévoilement», on parle de son " retrait », souvent aussi on use à son propos de l'expression "sans cesse », mais je ne vois, dans le texte d'Hésiode, aucun de ces termes apparaître en corrélation avec le Chaos.

L'interprétation du Chaos ici proposée, beaucoup moins tautégorique qu'il n'est dit, implique donc que cette entité primordiale n'est pas née ponctuellement aux yeux d'Hésiode, mais qu'elle est éternelle. L'A. reconnait que cette qualification n'est pas élaborée, mais qu'elle est implicite. Cette thèse, qui n'a pas été unanimement admise dans l'Antiquité, ne me parait pas indéfendable, mais je remarquerai que, sur la mythologie de l'origine et à s'en tenir au seul Hésiode, il resterait beaucoup à dire. On appréciera, en revanche, constituant l'essentiel du chapitre, les développements consacrés à la notion d'éternité chez les premiers philosophes, en corrélation avec des notions capitales comme aiôn, genesis, phusis. La conclusion clôt cette revue par une évocation du vieux Phérécyde, le premier, semble-t-il, à avoir affirmé que les trois puissances primordiales qu'il promeut ont toujours été, et par des propos d'inspiration heideggérienne (on n'est pas très surpris). L'ouvrage comporte une bibliographie, et trois index, nominum, rerum et locorum.

André MOTTE

(Université de Liège)

Synnøve Des BOUVRIE (éd.), Myth and Symbol II. Symbolic phenomena in ancient Greek culture. Papers from the second and third international symposia on symbolism at the Norwegian Institute at Athens, September 21-24, 2000 and September 19-22, 2002, Bergen, 2004. 1 vol. $17,5 \times 24 \mathrm{~cm}, 390$ p. (Papers from the Norwegian Institute at Athens, 7). ISBN : 82-91626-22-7.

Ce volume présente une série de papiers présentés lors de deux colloques organisés à l'Institut norvégien d'Athènes. Un colloque sur le même thème, organisé à Tromsø, avait été publié en 2002. Avec son sous-titre, "Symbolic Phenomena in Ancient Greek Culture », il entendait guider l'étude du mythe grec vers les questions générales du symbolisme, dont les fondements méthodologiques étaient éclaircis dans une copieuse introduction de l'éditrice1.

${ }^{1}$ Voir le compte rendu dans Kernos 17 (2004), p. 331-333. 
Les contributions du présent volume puisent donc leur source dans un colloque intitulé "Cognitive and Affective Aspects of Symbols in Ancient Culture » et dans un autre sur "Their Occasion, Audience, and Performance in Ancient Greek Culture ». Dans l'introduction, l'éditrice annonce un déplacement du regard depuis le mythe comme forme de " pensée symbolique » vers le mythe comme forme d'« activité symbolique ». Cette inflexion fait également passer l'attention de la forme et du contenu vers les contextes et les fonctions des mythes. Les mythes sont donc conçus comme les véhicules pertinents des valeurs idéologiques ambiguës qui circulent dans une culture donnée, une vision en rapport avec les travaux récents dans les domaines plus larges de l'étude anthropologique, folklorique, littéraire et théorique de la mythologie. La plupart des contributeurs du volume sont des spécialistes des études classiques. Mais ils proviennent de différents domaines et de différents milieux (Suède, Hollande, France, Italie). Ils adoptent des méthodologies variées (comparative, structurale, historique, iconographique) et étudient un grand nombre de problèmes liés aux aspects culturels et aux processus symboliques impliqués dans les phénomènes langagiers, visuels et matériels associés au 'mythe'. D’autres périodes (étrusque, romaine, néo-classique), d'autres genres (légende, récit folklorique), et d'autres considérations théoriques sont aussi pris en compte. Le champ d'investigation et les objectifs de ce volume sont très vastes ${ }^{2}$, mais il n'y a guère de thème vraiment fédérateur ni de conclusion systématique. Aucun ordre particulier ne semble présider à l'organisation des différentes contributions. On les prendra donc dans leur ordre d'apparition en mettant en évidence les questions et les solutions mises en avant pour conceptualiser le 'mythe grec'.

Dans le premier article, W. Hansen pose la question suivante : "What moves a certain performer to sing a precise song on a given occasion and what does its story mean to the participants in the events? » Différents facteurs peuvent intervenir. À l'une des extrémités du spectre, le narrateur raconte une histoire parce qu'elle correspond adéquatement à une circonstance sociale. À l'autre extrémité, le narrateur peut adopter un motif traditionnel pour faire passer un sentiment personnel. Raconter oralement une histoire est dans l'un ou l'autre cas une action motivée socialement et psychologiquement. - M. Nielsen livre quant à elle de nouvelles interprétations de deux reliefs funéraires, l'un étrusque, l'autre grec, l'un de contenu narratif, l'autre non. N. se demande si ces scènes devaient être «lues » à plus d'un niveau symbolique. La représentation étrusque d'un mythe grec, 'affective' (symbolique), implique des questions cognitives de catégorisation et de structuration du monde. Le relief grec, peu 'mythique', bien que 'cognitif' (philosophique) en apparence, semble en fait induire des réponses affectives, associées au cycle de la vie et de la mort. Cela rend sans objet la discussion de la nature cognitive $v$ affective du mythe, ou du symbole et de la métaphore dans l'art ancien. - Chr. Auffarth traite de L'Assemblée des femmes d'Aristophane. Il donne de la profondeur aux processus cognitifs et affectifs qui sont conjointement à l'œuvre dans le 'rituel', le 'mythe' et le 'symbole' du renversement tel que la mise en scène de l'utopie des femmes prenant part à l'assemblée. La relation entre 'mythe' et 'réalité' dans les rituels d'inversion est paradoxale et complexe. Ces rituels présentent un monde transformé en utopie, qui est temporairement réel dans la performance, un monde qui ne semble pas limité par la réalité sociale. La totalité du processus et de l'expérience, toutefois, aura aussi un effet et un 'affect' sur la réalité sociale (le monde). - P. Ellinger livre dans un article la présentation du livre sur les «deux Pausanias » qu’il a publié depuis lors (cf. Kernos 19, 2006, p. 465467). - J. Henderson se tourne vers l'art néo-classique du XIXe s. et plus particulièrement vers la frise extérieure qui décore de Musée Thorvaldsen à Copenhague. Il montre comment la frise met en forme les thèmes mythiques et classiques en les adaptant aux préoccupations

2 Voir la table des matières dans Kernos 19 (2006), p. 505. 
du temps. - W. Hansen, une fois encore, explore comment les narrations modelées par les commentaires du narrateur et/ou de l'auteur (« embedded narration») peuvent contribuer à notre compréhension de leur signification individuelle et des processus de création et de transformation de ce sens. Il définit trois aspects : la résonance d'une histoire particulière (e.g. le récit d'Éros et Psyché chez Apulée); l'émergence, par la mise en évidence d'un point particulier à laquelle le narrateur/auteur veut aboutir (e.g. le récit sur l'orgueil d'Eurytion raconté à Ulysse par le roi Antinoos); la tension entre une histoire abstraite (dans un sens structural) et sa réalisation en diverses occasions (e.g. l'utilisation inattendue du mythe de Niobé par Achille à la fin de l'Iliade). - J. Bremmer se penche sur les contextes sociaux des récits mythiques, en choisissant deux situations 'genrées', les femmes à la maison et les hommes à la leschè. La documentation assez pauvre sur les histoires et mythes racontés par les femmes (e.g. Platon) montre l'importance accordée à la position sociale et à la segmentation des groupes: les récits des mères reçoivent une estime plus grande que ceux des vieilles femmes. Quant à la leschè, pour l'A., elle aurait rapidement perdu son importance politique et religieuse, en devenant un endroit où les vieillards se distraient avec des récits et des mythes divertissants, au contraire des mythes sérieux. Le statut et l'autorité attachés aux récits mythiques sont influencés par les facteurs sociaux et le contexte de la performance. Chr. Sourvinou-Inwood commence par définir le concept de mythe. Elle insiste sur les significations complexes des phénomènes en jeu et s'oppose aux approches réductrices dont il fait l'objet. Les mythes sont polysémiques. Pour réduire au maximum l'intrusion de filtres modernes, l'A. propose de reconstruire les schémas qui structurent les mythes. Chaque schéma apparaitra modifié dans des mythes différents et plusieurs schémas apparaittront structurant chaque mythe singulier. Ainsi, les paramètres de base par lesquels les Athéniens ont donné sens au mythe du rapt des Athéniennes par les Pélasges sont, par exemple, «la vulnérabilité de la communauté à travers ses femmes", "l'importance des fils légitimes ». Les schémas mythiques reflètent les catégories de propositions et articulent les réalités et les valeurs d'une société. - P. Vidal-Naquet compare les valeurs symboliques et mythiques du pays utopique de l'Atlantide et celles de la forteresse historique des Juifs à Massada, respectivement décrits par Platon et Flavius Josèphe. Le premier est mythique, mais Platon lui donne une consistance historique, tandis que la seconde est historique, mais acquiert une dimension mythique. Dans les deux cas, des processus narratifs, cognitifs et affectifs convergent pour constituer des symboles puissants, pour renforcer leur impact sur les perceptions de la nationalité et pour leur donner un pouvoir politique interculturel. Cela rend la distinction entre mythe et histoire peu opérante. - M. Skafte Jensen se fonde sur les travaux de l'anthropologue finois L. Honko, qui a étudié la récitation de l'épopée traditionnelle en Inde. La comparaison avec la récitation de l'épopée homérique est particulièrement éclairante. L'A. développe un modèle dynamique selon lequel les poètes 'oraux' ont un texte abstrait en tête et s'appuient sur un ensemble de motifs traditionnels et de lignes de récits. Chaque narration consiste en une formulation différente et s'adapte à un contexte particulier. Lorsqu'ils sont consignés, ces poèmes reflètent les processus de la performance et ceux de l'écriture, ce qui complique davantage les choses. - V. Masciadri creuse la question de la 'structure profonde' du mythe en comparant le récit du crime des Lemniennes, celui de l'enlèvement des Athéniennes par les Pélasges et le mythe des Danaïdes. Il s'agit chaque fois de l'histoire d'un massacre conduit par un groupe de femmes. Les différences sont structurées par des oppositions binaires «à la Lévi-Strauss ». - D. Jordan discute les représentations du sacrifice préparatoire à l'initiation aux mystères d'Éleusis en convoquant l'urne romaine de Lovatelli, les Nuées d'Aristophane, le Protagoras et le Phèdre de Platon, et même la fable de la Jeune fille et l'oie des frères Grimm, ce qui élargit notre compréhension de la complexité de l'art narratif et de la transmission. - H. Whittaker observe que les plongeons ont joué un rôle dans le symbolisme funéraire de la Grèce 
archaïque comme de la Rome impériale, ainsi qu'en d'autres endroits et à d'autres époques. L'argument privilégié est celui des contacts, des influences et des continuités. Mais il pourrait aussi s'agir de l'illustration d'une idée universelle. - J. Stroszeck se penche sur les trophées. Des tropaia étaient érigés après une victoire au combat. L'A. prend en compte la variété de leurs caractéristiques et des endroits où ils étaient élevés. Des objets matériels pouvaient servir de symboles dans le sens où ils étaient porteurs de connotations affectives et de significations cognitives en réponse à des besoins individuels ou collectifs dans des situations spécifiques. - L. Bruit Zaidman montre qu'il faut rendre à l'Hippolyte d'Euripide sa juste dimension religieuse dans le contexte auquel il appartient, sans projeter sur la tragédie une opposition indue entre chasteté - qui serait représentée par Artémis - et luxure - qui serait représentée par Aphrodite. - S. Des Bouvrie adopte une théorie anthropologique du symbolisme en se concentrant sur la panégyrie d'Olympie, pour examiner la nature de célébration de la récitation des épinicies. Elle montre comment, durant ce processus, des catégories et des symboles 'synthétiques' créent et sont créés - en ayant dans le cas d'Olympie une relation particulière à l'art militaire et à l'idéologie hoplitique du héros mâle. Le symbolisme n'est pas purement inconscient et passif.

Beaucoup de contributions de ce volume sont de grande qualité. Elles seront instructives et stimulantes pour des chercheurs travaillant dans de nombreux domaines. L'idée centrale qu'il convient d'intégrer les 'symboles' et les 'mythes' dans nos recherches historiques et ne pas les considérer en opposition avec celles-ci est un point de vue utile et rafraîchissant. Malheureusement, il subsiste d'autres dichotomies rigides comme celle qui oppose 'mythe/affectif' vs 'philosophique/cognitif. De plus, la définition et l'usage du concept de 'symbole' et de celui de 'phénomène symbolique' restent peu clairs et ne font l'objet d'aucune conclusion. Le volume en tant que tel est moins réussi que les contributions particulières qu'il contient. En cela aussi, il reflète la richesse et la complexité du sujet dont il traite.

Ellen VAN KEER

(VUB - Centre Leo Apostel)

BARRA-SALZÉDO Edoarda, En soufflant la grâce. Âmes, souffles et bumeurs en Grèce ancienne, Grenoble, Jérôme Millon, 2007. 1 vol. $16 \times 24$ cm, 246 p. (coll. Horos). ISBN : 978-2-84137-207-2.

Au début du XIXe siècle, E. Bethe faisait l'hypothèse que les relations sexuelles entre un éraste et son éromène étaient vues comme le vecteur du transfert de la «vertu » de l'amant vers son aimé, et que le véhicule de cet âme vertueuse était le sperme. Un passage d'Élien venait soutenir cette argumentation puisqu'il attestait qu'à Sparte, les jeunes demandaient aux aînés de les «inspirer", ce qui signifie " aimer» (V.H. III, 12). Le transfert " pneumatique » de la vertu était lié à la semence. Une telle hypothèse a été combattue par ceux qui ont étudié l'homosexualité grecque et l'A. fait le point sur la question en introduction. Elle montre également que, si l'Esprit saint de la tradition catholique a pu constituer un obstacle à l'étude du pneuma grec dans les représentations religieuses, il n'en reste pas moins que l'on retrouve, à la fois dans le corpus hippocratique et chez les Pères de l'Église, la dimension pneumatique de la semence. Ce livre a donc l'ambition de comprendre comment les différents témoignages qui associent souffle, âme et sperme s’inscrivent «dans un système symbolique très complexe où le pneuma, le plus 'spirituel' des éléments matériels, constitue le principe dont l'âme est issue » (p. 11).

De démonstration à cette fin, il ne sera pas question. La méthode expositive privilégiée par l'A. relève de la juxtaposition des témoignages et de leur mise en série dont surgissent des questions, tout d'abord, des réponses, ensuite, dont l'élément fédérateur est le pneuma. 